The formation of electrostatic shocks in quantum plasmas with relativistically degenerate electrons

This article has been downloaded from IOPscience. Please scroll down to see the full text article.

2012 EPL 9715001

(http://iopscience.iop.org/0295-5075/97/1/15001)

View the table of contents for this issue, or go to the journal homepage for more

Download details:

IP Address: 130.159.82.179

The article was downloaded on 10/09/2013 at 16:26

Please note that terms and conditions apply. 


\title{
The formation of electrostatic shocks in quantum plasmas with relativistically degenerate electrons
}

\author{
B. Eliasson ${ }^{1(a)}$ and P. K. Shukla ${ }^{2,3(b)}$ \\ ${ }^{1}$ Institut für Theoretische Physik, Fakultät für Physik und Astronomie, Ruhr-Universität Bochum \\ D-44780 Bochum, Germany, EU \\ ${ }^{2}$ RUB International Chair, International Centre for Advanced Studies in Physical Sciences, \\ Fakultät für Physik und Astronomie, Ruhr-Universität Bochum - D-44780 Bochum, Germany, EU \\ ${ }^{3}$ Department of Mechanical and Aerospace Engineering, University of California, San Diego - La Jolla, \\ CA 92093, USA
}

received 25 October 2011; accepted 16 November 2011

published online 29 November 2011

PACS 52.35.Tc - Shock waves and discontinuities

PACS 52.35.Fp - Electrostatic waves and oscillations (e.g., ion-acoustic waves)

PACS 97.20.Rp - Faint blue stars (including blue stragglers), white dwarfs, degenerate stars, nuclei of planetary nebulae

\begin{abstract}
The formation of electrostatic shocks in a super-dense plasma composed of relativistically degenerate electrons and fully ionized ions is theoretically investigated. We find analytic solutions in the form of simple waves and derive expressions for shock speeds in limiting cases. The theory has applications to large-amplitude acoustic waves excited in white dwarf stars due to dramatic events such as collision with other astrophysical bodies or supernova explosions.
\end{abstract}

Copyright (C) EPLA, 2012

Introduction. - White dwarf stars are examples of matter under extreme conditions [1-4]. They constitute the end product of collapsed stars, where the thermal pressure no longer can sustain the gravitational force. They evolve from extremely hot early stages where the energy loss is mainly due to neutrino fluxes, to cooler stages dominated by photon radiation. The core of white dwarfs typically consists of fully ionized helium, carbon and oxygen with an outer shell of hydrogen. One of the most intriguing quantum phenomena is the structure of white dwarf stars, which couples the degeneracy of electrons due to the Pauli exclusion principle and Heisenberg's uncertainty principle to the stability of the white dwarf star on macroscopic scales via the balance between gravitational pull and the degeneracy pressure of the electrons. When the Fermi energy of the electrons becomes comparable to or higher than the electron rest energy, relativistic effects come into play and the equation of state changes from $P \sim n^{5 / 3}$ to $P \sim n^{4 / 3}$, which makes the white dwarf star gravitationally unstable for masses larger than about 1.4 solar masses [1-6].

\footnotetext{
(a) E-mail: bengt@tp4.rub.de

(b) E-mail: profshukla@yahoo.de
}

The observation of white dwarf stars is mainly due to their electromagnetic radiation, which reveals their physical properties and dynamics [7]. There are about two hundred observations of pulsating white dwarf stars so far. The pulsation period typically falls in the range from 2 to 35 minutes and can be attributed to nonradial gravity ( $g$-mode) oscillation modes. The observations and theory of these pulsations is now well established, and the discipline of white-dwarf asteroseismology is used to study their rotation period, mass, equation of state, etc. $[8,9]$. In addition to the gravity waves, the theory also predicts the existence of acoustic modes ( $p$-modes) where the ions provide the inertia and mainly the electron degeneracy pressure provides the restoring force. Typical oscillation periods of globally propagating $p$-modes is set by the time for the wave to travel across the star and lies in the range of a few seconds, two orders of magnitude shorter than $g$-mode oscillations. These modes were early predicted [10], but are yet to be observed [11].

The lack of observations does not necessarily mean that the $p$-mode oscillations are not excited, but since the $p$-mode wave acts primarily in the vertical direction, the huge gravity may lead to an associated vertical motion that is below the detection limit. On the other hand, detectable, large-amplitude acoustic waves could be 
excited in extreme events, such as supernova explosions at the outer shells of the star or during collisions between the white dwarf with other astrophysical bodies. When the amplitudes of the oscillations are large, nonlinear effects must be taken into account. The nonlinearities can lead to self-steepening and shock formation, similar to hydrodynamic shocks in gases and plasmas. Our aim here is to present a simple theory for the self-steepening and shock formation of the matter in the interior of white dwarf stars and derive expressions for the nonlinear wave and shock speeds for varying degrees of relativistic electron degeneracy.

Mathematical model. - The dynamic model for the fully ionized ions (e.g., carbon or oxygen) is governed by the continuity equation

$$
\frac{\partial n_{i}}{\partial t}+\frac{\partial\left(n_{i} v_{i}\right)}{\partial x}=0
$$

and the momentum equation

$$
\frac{\partial v_{i}}{\partial t}+v_{i} \frac{\partial v_{i}}{\partial x}=\frac{Z_{i} e E}{m_{i}}
$$

where $Z_{i}$ is the charge state of the nondegenerate ions. In the interior of the white dwarf stars, the ions are fully ionized due to pressure ionization, and hence $Z_{i}$ equals the number of protons in the ion nucleus. It should be noted that for most ions, like helium, carbon and oxygen, there is an almost equal amount of neutrons and protons in the nucleus, where the neutrons and protons have approximately equal mass, so that the ratio $Z_{i} / m_{i} \approx$ $1 /\left(2 m_{p}\right)$, where $m_{p}$ is the proton mass. The exception is hydrogen in the outer shell of the star which only has a proton in the nucleus and hence $Z_{i} / m_{i}=1 / m_{p}$. On the other hand, it is believed that more neutron-rich iron exists in the center of the white dwarf stars, with $Z_{i} / m_{i}<$ $1 /\left(2 m_{p}\right)$. We here consider oscillation spatial scales much smaller than the size of the star, and therefore the gravitational force directed downwards is cancelled exactly by an upward directed electrostatic force on the ions. For large-scale global modes, the variability of the density and gravity must be taken into account. Furthermore, eq. (2) is valid as long as the wave period is much larger than the time scale for the ion correlations as well as the work frequency is much larger than the damping rate caused by the ion fluid shear viscosity [12-14].

The degenerate electrons can be considered inertialess and are governed by the force balance equation

$$
0=-e E-\frac{1}{n_{e}} \frac{\partial P_{e}}{\partial x}
$$

where the relativistic electron degeneracy pressure $P_{e}$ is given by $[1-4]$

$$
P_{e}=\frac{\pi m_{e}^{4} c^{5}}{3 h^{3}}\left[\xi\left(2 \xi^{2}-3\right)\left(\xi^{2}+1\right)^{1 / 2}+3 \operatorname{arcsinh} \xi\right]
$$

where $m_{e}$ is the electron mass, $c$ the speed of light in vacuum, and $h$ Planck's constant. We have denoted

$$
\xi=\frac{p_{0}}{m_{e} c}
$$

where

$$
p_{0}=\left(\frac{3 h^{3} n_{e}}{8 \pi}\right)^{1 / 3}
$$

is the momentum of the fastest electrons in the electron distribution function (at the Fermi surface). It is convenient to combine eqs. (5) and (6) to have

$$
\xi=\lambda_{C} n_{e}^{1 / 3}
$$

where we introduced the scaled Compton length

$$
\lambda_{C}=\left(\frac{3}{8 \pi}\right)^{1 / 3} \frac{h}{m_{e} c} .
$$

The value of $\xi$ determines the relativistic effects. When the mean inter-electron distance $n_{e}^{-1 / 3}$ is much smaller than the Compton length, so that $\xi \gg 1$, then the pressure is in the ultra-relativistic regime. On the other hand, for $\xi \ll 1$, the pressure equals the non-relativistic electron degeneracy pressure.

Using the quasi-neutrality condition, viz. $Z_{i} n_{i}=n_{e} \equiv n$, in eq. (1), and eq. (3) to eliminate $E$ in eq. (2), we have the continuity equation

$$
\frac{\partial n_{i}}{\partial t}+\frac{\partial\left(n_{i} v_{i}\right)}{\partial x}=0
$$

and the momentum equation

$$
\frac{\partial v_{i}}{\partial t}+v_{i} \frac{\partial v_{i}}{\partial x}=-\frac{Z_{i}}{m_{i} n} \frac{\partial P_{e}}{\partial x}=-\frac{Z_{i} P_{e}^{\prime}(n)}{m_{i} n} \frac{\partial n}{\partial x} .
$$

Here the prime denotes differentiation with respect to $n$, and

$$
P_{e}^{\prime}(n)=\left(\frac{3}{8 \pi}\right)^{2 / 3} \frac{h^{2} n^{2 / 3}}{3 m_{e} \gamma}=\frac{n^{2 / 3} m_{e} v_{F e}^{2}}{3 n_{0}^{2 / 3} \gamma},
$$

where

$$
\gamma=\sqrt{1+\lambda_{C}^{2} n^{2 / 3}}
$$

is the relativistic gamma factor for the electrons on the Fermi surface, $v_{F e}=\hbar\left(3 \pi^{2} n_{0}\right)^{1 / 3} / m_{e}$ is the nonrelativistic expression for the electron Fermi speed, $\hbar=h / 2 \pi$ the reduced Planck's constant, and $n_{0}$ is the equilibrium electron number density where the plasma is at rest. The nonrelativistic Fermi speed does not correspond to the real speed of the electrons in the relativistic case, but is introduced for convenience. In the ultra-relativistic limit $\lambda_{C}^{2} n_{0}^{2 / 3} \gg 1$, we in fact also have $v_{F e}>c$. We note that using eq. (11) into eq. (10) yields

$$
\frac{\partial v_{i}}{\partial t}+v_{i} \frac{\partial v_{i}}{\partial x}=-Z_{i} c^{2} \frac{m_{e}}{m_{i}} \frac{\partial \gamma}{\partial x}
$$


while using eq. (11) into eq. (3), we have

$$
0=-e E-m_{e} c^{2} \frac{\partial \gamma}{\partial x}
$$

We note that the density fluctuations are directly associated with an electrostatic potential $\phi$, which is obtained by using $E=-\partial \phi / \partial x$ in eq. (14). After integration in space we have

$$
\phi=\frac{m_{e} c^{2}}{e}\left[\gamma(n)-\gamma\left(n_{0}\right)\right] .
$$

The electrostatic potential glues the ion fluid to the degenerate electrons and keeps the plasma quasi-neutral.

In order to find the nonlinear wave speeds, we diagonalize the system of equations (9) and (10). We therefore write them in the matrix form as

$$
\frac{\partial}{\partial t}\left[\begin{array}{l}
n \\
v_{i}
\end{array}\right]+\left[\begin{array}{cc}
v_{i} & n \\
\frac{Z_{i} P_{e}^{\prime}(n)}{m_{i} n} & v_{i}
\end{array}\right] \frac{\partial}{\partial x}\left[\begin{array}{l}
n \\
v_{i}
\end{array}\right]=\left[\begin{array}{l}
0 \\
0
\end{array}\right] .
$$

Denoting the square matrix in the left-hand side by $A$, the nonlinear ion acoustic wave speed is found from the eigenvalues $u$ of $\mathrm{A}$, or

$$
\operatorname{det}(\mathrm{A}-u \mathrm{I})=\left(v_{i}-u\right)^{2}-\frac{Z_{i} P_{e}^{\prime}(n)}{m_{i}}=0,
$$

where I denotes the unit matrix. Solving for $u$, we have the solutions

$$
u_{ \pm}=v_{i} \pm \sqrt{\frac{Z_{i} P_{e}^{\prime}(n)}{m_{i}}} .
$$

Using the methods from linear algebra, we construct a diagonalizing matrix $C$ where the columns are the eigenvectors of $\mathrm{A}$. The eigenvectors $X_{ \pm}$are found from the solutions of the equation $\left(\mathrm{A}-u_{ \pm} \mathrm{I}\right) X_{ \pm}=0$, from which we have the diagonalizing matrix

$$
\mathrm{C}=\left[X_{+} X_{-}\right]=\left[\begin{array}{cc}
n \sqrt{\frac{m_{i}}{Z_{i} P_{e}^{\prime}(n)}} & -n \sqrt{\frac{m_{i}}{Z_{i} P_{e}^{\prime}(n)}} \\
1 & 1
\end{array}\right],
$$

and its inverse

$$
\mathrm{C}^{-1}=\left[\begin{array}{cc}
\frac{1}{2 n} \sqrt{\frac{Z_{i} P_{e}^{\prime}(n)}{m_{i}}} & \frac{1}{2} \\
-\frac{1}{2 n} \sqrt{\frac{Z_{i} P_{e}^{\prime}(n)}{m_{i}}} & \frac{1}{2}
\end{array}\right] .
$$

We now multiply eq. (16) from the left by $\mathrm{C}^{-1}$ and use

$$
\mathrm{C}^{-1} \mathrm{AC}=\left[\begin{array}{cc}
u_{+} & 0 \\
0 & u_{-}
\end{array}\right]
$$

to obtain the new, diagonalized system of equations

$$
\begin{gathered}
\frac{\partial \psi_{+}}{\partial t}+u_{+} \frac{\partial \psi_{+}}{\partial x}=0 \\
\frac{\partial \psi_{-}}{\partial t}+u_{-} \frac{\partial \psi_{-}}{\partial x}=0
\end{gathered}
$$

where the new unknowns are

$$
\psi_{ \pm}=\frac{v_{i}}{2} \pm \int_{n_{0}}^{n} \frac{1}{2 n} \sqrt{\frac{Z_{i} P_{e}^{\prime}(n)}{m_{i}}} \mathrm{~d} n .
$$

In general, $\psi_{+}$denotes a wave propagating in the positive $x$-direction and $\psi_{-}$a wave propagating in the opposite direction. Simple wave solutions are found by setting either $\psi_{+}$or $\psi_{-}$to zero. Setting $\psi_{-}$to zero, we find

$$
v_{i}=\int_{n_{0}}^{n} \frac{1}{n} \sqrt{\frac{Z_{i} P_{e}^{\prime}(n)}{m_{i}}} \mathrm{~d} n
$$

and the nonlinear wave speed is

$$
u_{+}=\int_{n_{0}}^{n} \frac{1}{n} \sqrt{\frac{Z_{i} P_{e}^{\prime}(n)}{m_{i}}} \mathrm{~d} n+\sqrt{\frac{Z_{i} P_{e}^{\prime}(n)}{m_{i}}} \equiv u(n)
$$

and the unknown

$$
\psi_{+}(n)=\int_{n_{0}}^{n} \frac{1}{n} \sqrt{\frac{Z_{i} P_{e}^{\prime}(n)}{m_{i}}} \mathrm{~d} n .
$$

Since $\psi_{+}$depends on $n$ only, the chain rule can be used in the time and space derivatives in eq. (22) to obtain (after eliminating the common factor $\left.\psi_{+}^{\prime}(n)\right)$

$$
\frac{\partial n}{\partial t}+u(n) \frac{\partial n}{\partial x}=0
$$

The linear ion acoustic speed $C_{s}$ is obtained by setting $n=n_{0}$ in eq. (26). We then have, using eq. (11) for $P_{e}^{\prime}(n)$

$$
C_{s} \equiv u\left(n_{0}\right)=V_{F e} \sqrt{\frac{Z_{i} m_{e}}{3 m_{i} \gamma_{0}}},
$$

where $\gamma_{0}=\sqrt{1+\lambda_{C}^{2} n_{0}^{2 / 3}}$. For the nonlinear case, eq. (28) has the formal solution $n=N_{0}[x-u(n) t]$, where $N_{0}$ is a function of one argument determined by the initial condition for $n$ at $t=0$. This solution is valid only as long as $n$ is differentiable. Due to the nonlinear wave speed, the solution can self-steepen and develop discontinuities, or shocks, after a finite time, even if the initial condition is smooth. Due to the conservation of mass and momentum, the shock speed is given by the Rankine-Hugoniot condition

$$
v_{\text {shock }}=\frac{F\left(n_{L}\right)-F\left(n_{R}\right)}{n_{L}-n_{R}},
$$

where $n_{L}$ and $n_{R}$ are the values of $n$ on the left and right side of the shock, respectively, and $F(n)=\int_{n_{0}}^{n} u \mathrm{~d} n$ is the flux function. Below, we will use $n_{R}=n_{0}$ and $n_{L}>n_{0}$, i.e. the high density shock is propagating into the equilibrium matter. In the small amplitude limit, $n_{L} \rightarrow n_{R}=n_{0}$, the shock speed equals the linear ion acoustic speed $C_{s}$.

The nonlinear wave speed can for the general case only be expressed in terms of special functions or evaluated 
numerically. However, for the limiting nonrelativistic and ultrarelativistic cases, simple expressions can be derived. In the nonrelativistic limit $\lambda_{C}^{2} n_{0}^{2 / 3} \ll 1$, we have $\gamma=1$ and

$$
P_{e}^{\prime}(n)=\frac{n^{2 / 3} m_{e} v_{F e}^{2}}{3 n_{0}^{2 / 3}},
$$

and we can readily carry out the integral in eq. (26) to obtain the nonlinear wave speed

$$
u=\sqrt{\frac{Z_{i} m_{e}}{3 m_{i}}}\left(\frac{4 n^{1 / 3}}{n_{0}^{1 / 3}}-3\right) v_{F e} .
$$

For this case we obtain from eq. (30) the shock speed

$$
v_{\text {shock }}=3 \sqrt{\frac{Z_{i} m_{e}}{3 m_{i}}} \frac{n_{L}}{n_{0}} \frac{\left(n_{L}^{1 / 3} / n_{0}^{1 / 3}-1\right)}{\left(n_{L} / n_{0}-1\right)} v_{F e} .
$$

On the other hand, in the ultra-relativistic limit $\lambda_{C}^{2} n_{0}^{2 / 3} \gg 1$, we have $\gamma=\lambda_{C} n^{1 / 3}$, and

$$
P_{e}^{\prime}(n)=\frac{c \hbar\left(3 \pi^{2} n\right)^{1 / 3}}{3}
$$

For this case, we obtain the nonlinear wave speed

$$
u=\sqrt{\frac{Z_{i} c \hbar n_{0}^{1 / 3}}{3 m_{i}}}\left(\frac{7 n^{1 / 6}}{n_{0}^{1 / 6}}-6\right)\left(3 \pi^{2}\right)^{1 / 6}
$$

and the shock speed

$$
v_{\text {shock }}=6 \sqrt{\frac{Z_{i} c \hbar n_{0}^{1 / 3}}{3 m_{i}}} \frac{n_{L}}{n_{0}} \frac{\left(n_{L}^{1 / 6} / n_{0}^{1 / 6}-1\right)}{\left(n_{L} / n_{0}-1\right)}\left(3 \pi^{2}\right)^{1 / 6} .
$$

Let us compare the linear and nonlinear wave speeds for parameters that are representative of nonrelativistic and ultra-relativistic degenerate electrons. We consider fully ionized ions so that $Z_{i} / m_{i}=1 /\left(2 m_{p}\right)$. For a nonrelativistically large density $n_{0}=10^{34} \mathrm{~m}^{-3}$, we have the linear wave speed $C_{s}=7.3 \times 10^{5} \mathrm{~m} / \mathrm{s}$, while for a four orders of magnitude larger, ultra-relativistically large density $n_{0}=10^{38} \mathrm{~m}^{-3}$, the linear wave speed increases by one order of magnitude to $C_{s}=6.7 \times 10^{6} \mathrm{~m} / \mathrm{s}$. We note that the linear wave speed is proportional to $n_{0}^{1 / 3}$ in the nonrelativistic case and to $n_{0}^{1 / 6}$ in the ultra-relativistic case. Next, we consider the nonlinear wave propagation, where the wave amplitudes $n$ and $n_{L}$ of the density are taken to be twice that of the ambient value $n_{0}$. For the nonrelativistic case, we then have a nonlinear wave speed $u=1.49 \times 10^{6} \mathrm{~m} / \mathrm{s}$, and a shock speed $v_{\text {shock }}=$ $1.14 \times 10^{6} \mathrm{~m} / \mathrm{s}$. As expected, the shock speed is larger than the linear wave speed, but somewhat smaller than the nonlinear wave speed. For the ultra-relativistic case, we have the nonlinear wave speed $u=1.25 \times 10^{7} \mathrm{~m} / \mathrm{s}$, and the shock speed $v_{\text {shock }}=1.0 \times 10^{7} \mathrm{~m} / \mathrm{s}$. Hence, for both the nonrelativistic and ultra-relativistic cases, the nonlinear wave speeds are significantly increased for larger amplitudes of the nonlinear waves and shocks.

Conclusions. - We have here presented a theory for the self-steepening and shock formation of the super-compressed matter in white dwarf stars. For large-amplitude acoustic-like waves, the ions provide the inertia and the electron degeneracy pressure provides the restoring force. We have found simple wave solutions that reveal the nonlinear wave speed of the shocks, and simplified expressions are derived in the limiting cases of nonrelativistic and ultra-relativistic electron degeneracy. The theory presented here could be used as building blocks for global nonlinear models of white dwarf stars, where the inhomogeneity of the density and gravitational field must be taken into account. The theory could have relevance for acoustic waves following dramatic events, such as collisions between white dwarf stars and other astrophysical bodies as well as for supernova explosions [15,16]. Finally, the present investigation can be readily generalized to include an ambient magnetic field, in which we have the possibility of magnetosonic solitons [17] and shocks [18] in dense plasma.

This research was supported by the Deutsche Forschungsgemeinschaft (DFG), Bonn, through the project SH21/3-2 of the Research Unit 1048.

\section{REFERENCES}

[1] Chandrasekhar S., Philos. Mag. (Suppl.), 11 (1931) 592.

[2] Chandrasekhar S., Astrophys. J., 74 (1931) 81.

[3] Chandrasekhar S., Mon. Not. R. Astron. Soc., 95 (1935) 207.

[4] Chandrasekhar S., An Introduction to the Study of Stellar Structure (Dover, New York) 1939, p. 412.

[5] Balberg S. and Shapiro S. L., The Properties of Condensed Matter in White Dwarfs and Neutron Stars, in Levy M., Bass H., Stern R. and Paspet R. (Editors), Handbook of Elastic Properties of Solids, Liquids, and Gases, Vol. IV (Academic Press, UK) 2000 Chapt. 7, arXiv:astro-ph/0004317v2.

[6] Fontaine G. and Wesemae F., White Dwarfs, in Murdin P. (Editor), Encyclopedia of Astronomy and Astrophysics (Nature Publishing Group, New York, Tokyo) 2001, p. 3525.

[7] Shapiro S. I. and Teukolsky S. A., Black Holes, White Dwarfs, and Neutron Stars: The Physics of Compact Objects (Wiley, New York) 1983.

[8] Winget D. E. and Kepler S. O., Annu. Rev. Astron. Astrophys., 46 (2008) 157.

[9] Fontaine G. and Brassard P., Publ. Astron. Soc. Pac., 120 (2008) 1043.

[10] Ostriker J. P., Annu. Rev. Astron. Astrophys., 9 (1971) 535 . 
[11] Silvotti R., Fontaine G. and Pavlov M. et al., Astron. Astrophys., 525 (2011) A64.

[12] Shukla P. K. and Eliasson B., Rev. Mod. Phys., 83 (2011) 885.

[13] Mithen J. P., Daligault J. and Gregori G., Phys. Rev. E, 83 (2011) 105401(R).

[14] Shukla P. K., Mamun A. A. and Mendis D. A., Phys. Rev. E, 84 (2011) 026405.
[15] Casanova J., José J., García-Berro E., Shore S. N. and CAlder A. C., Nature (London), 478 (2011) 490, doi:10.1038/nature10520.

[16] Fortov V. E., Phys. Usp., 52 (2009) 615.

[17] Marklund M., Eliasson B. and Shukla P. K., Phys. Rev. E, 76 (2007) 067401.

[18] Shukla P. K., Eliasson B. and Stenflo L., EPL, 95 (2011) 045001. 